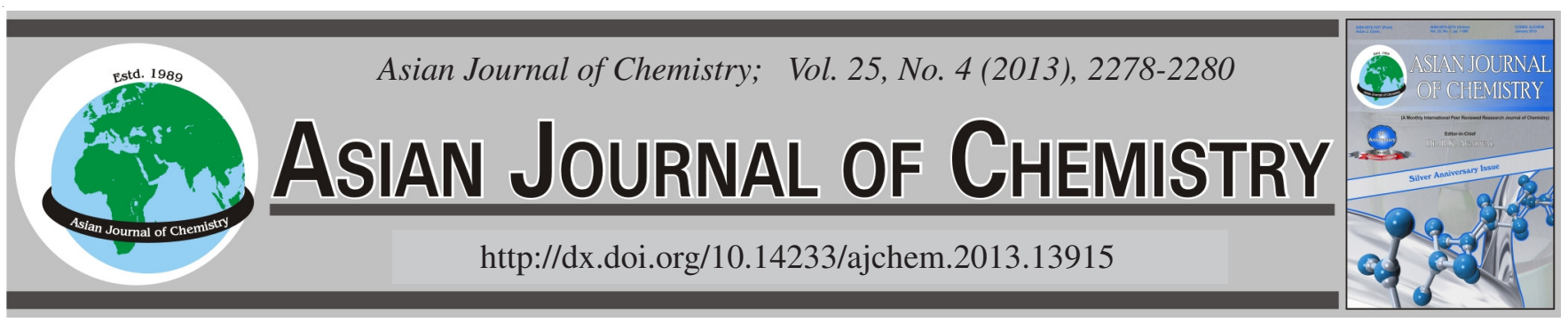

\title{
Fabrication and Efficient Acidichromism of Langmuir-Blodgett Films of Azo Group-Modified Phthalocyanines
}

JIANQIANG LI

Department of Industrial Engineering, Dongying Vocational College, Dongying 257091, P.R. China

Corresponding author: E-mail: dyzyxyljq@126.com

(Received: 16 April 2012;

Accepted: 22 October 2012)

AJC-12330

Langmuir-Blodgett thin films of the azo group-modified phthalocyanines are successfully prepared. The aggregated and acidichromic properties of Langmuir-Blodgett films has been investigated.

Key Words: Langmuir-Blodgett films, Fabrication, Acidichromism, Phthalocyanines.

\section{INTRODUCTION}

Phthalocyanines (Pcs) are an important class of pigments that have fascinated scientists for many years because of their applications in various disciplines ${ }^{1}$. A wide range of methods have recently been reported for modifying the supramolecular architecture of aggregated dyes such as the symmetrical phthalocyanines in both solutions and thin films formation ${ }^{2-8}$. Langmuir-Blodgett (LB) thin film deposition or solution casting strategies are made possible by side chain modifications ${ }^{8}$.

We have reported on the preparation and spectral property of the azo group-modified phthalocyanines containing shorter $\mathrm{C}_{2}$-alkyl chains on the periphery ${ }^{9}$. In order to have further studies of this class of azo group-modified phthalocyanines, we here present two new phthalocyanines with longer $\mathrm{C}_{6}$-alkyl chains on the periphery, tetrakis $[4-(\mathrm{N}, \mathrm{N}-$ dihexylamino $)$ phenylazo]phthal ocyaninato copper (compound a) and tetrakis [4-(N,N-dihexylamino)phenylazo]phthalocyanine (compound b) (Scheme-I).

\section{EXPERIMENTAL}

Tetrakis[4-(N,N-dihexylamino) phenylazo]phthal ocyaninato copper (compound a) and tetrakis [4-(N,N-dihexylamino)phenylazo]phthalocyanine (compound b) prepared following our procedure described previously ${ }^{9}$. Both of them exhibit excellent solubility in most organic solvents, such as dichloromethane, chloroform, THF and the like, which makes possible the fabrication of their Langmuir-Blodgett films and to have further study of the behaviour of their solid state.

\section{RESULTS AND DISCUSSION}

Fig. 1 shows the surface pressure-area isotherms for a (solid line) and for $\mathbf{b}$ (dashed line) in the preparation of

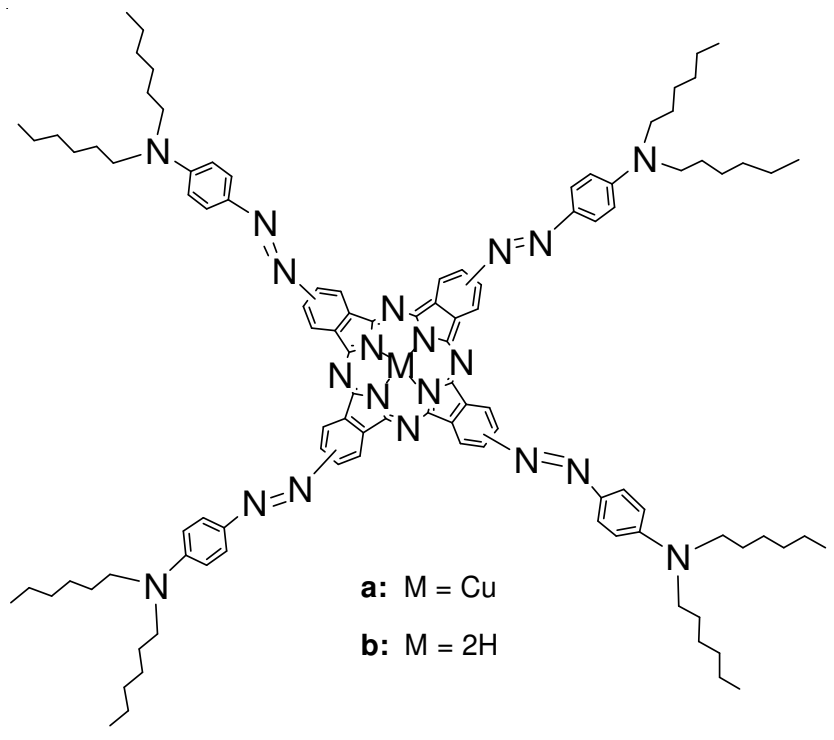

Scheme-I

Langmuir-Blodgett thin films. Extrapolation of the linear part of the isotherms to zero pressure give the limiting molecular areas of 1.05 and $1.02 \mathrm{~nm}^{2}$ per molecule for $\mathrm{a}$ and $\mathrm{b}$, respectively. Based on the SEM measurement of the molecule structure, the radius of the molecular structure from the molecular center to the most far periphery nitrogen atom is about $1.02 \mathrm{~nm}$. And considering of the $\mathrm{C}_{6}$-alkyl chains on the periphery, the plane of the phthalocyanines cannot be completely parallel to the water surface, they should be stacked in edge-on arrangement on the water surface. The limiting molecular area of metalphthalocyanine is larger than that of the metal-free phthalocyanine which is similar to literature reports ${ }^{10}$. 


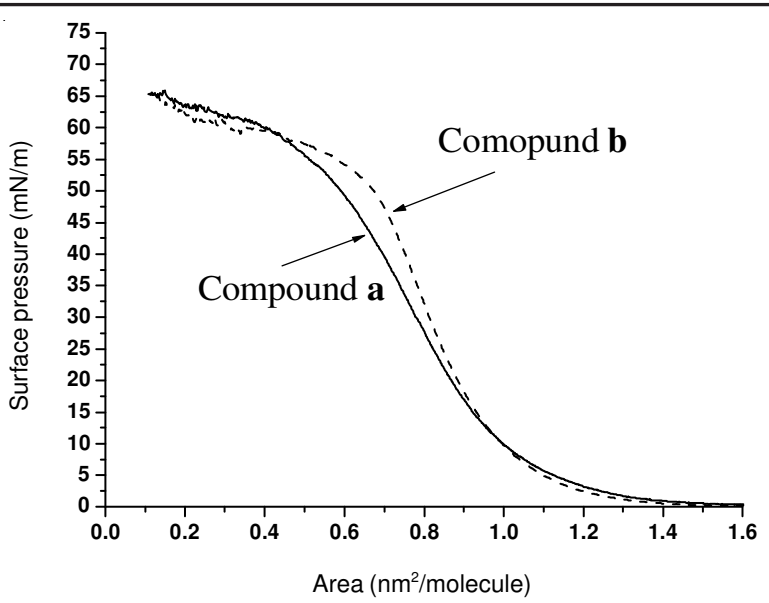

Fig. 1

These Langmuir-Blodgett thin films were found to show a highly efficient acidichromic property. In the case of Langmuir-Blodgett films, the degree of acidichromism can be controlled by treatment of them with gaseous $\mathrm{HCl}$ and $\mathrm{NH}_{3}$, alternately. Upon exposure of the Langmuir-Blodgett film to $\mathrm{HCl}$ gas, its colour turned purple rapidly from brown. An acidichromic transition for the Langmuir-Blodgett thin film of a is shown in Fig. 2. Line 1 is the absorption spectrum of the 20-layer Langmuir-Blodgett film deposited on the substrate of quartz under an average surface-pressure of $30 \mathrm{mN}$. After being subjected to gaseous $\mathrm{HCl}$ over $5 \mathrm{~s}$, the colour of the thin film changed thoroughly from brown to purple and the absorption spectrum of the acidified thin film shows a significant change (line 2): the absorption peak at $407 \mathrm{~nm}$ becomes an absorption valley and a new absorption peak appears at $551 \mathrm{~nm}$. The red-shift of the Q-band, though smaller, can also be discerned. Lines 3-9 demonstrate a gradual transformation of the absorption spectra of the acidified thin film when it stood under ambient conditions and lost $\mathrm{HCl}$ molecules slowly. Finally the spectrum of the acidichromic Langmuir-Blodgett thin film returned to its original pattern. And also, such a recovery process can be accelerated by exposure of the acidified Langmuir-Blodgett film to gaseous $\mathrm{NH}_{3}$. In addition, such a cycle of colour change can be repeated many times by treatment of the Langmuir-Blodgett film with $\mathrm{HCl}$ and $\mathrm{NH}_{3}$, alternately. For instant, 20 cycles were realized for a 20-layer Langmuir-Blodgett film of a, as shown in Fig. 2. These results confirmed that this acidichromism is a reversible process.

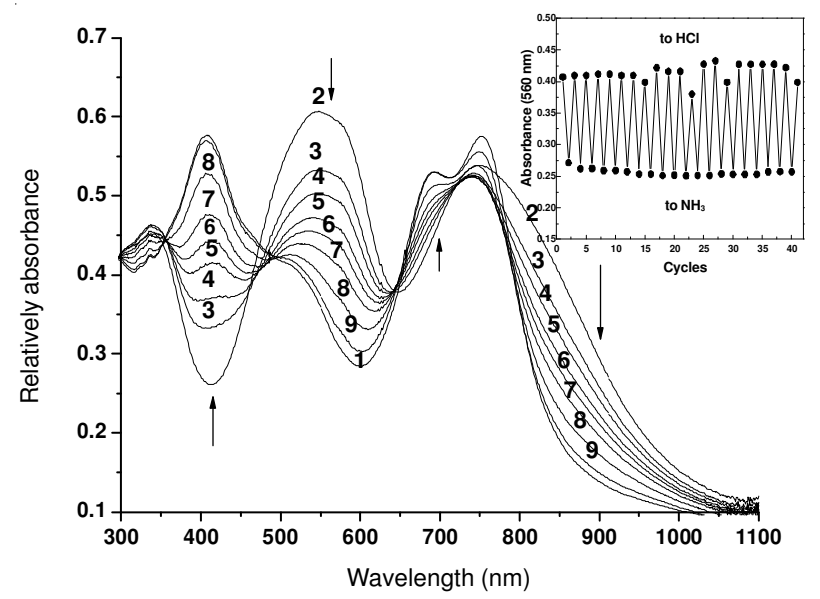

Fig. 2. UV-visible absorption spectra of the Langmuir-Blodgett thin film of compound a showing the reversible acidichromism process

This property could result from rearrangement of the molecular structure of the prolonged conjugated $\pi$ system induced by a proton-acidifying process. The acidichromic process may be follow the mechanism as show in Scheme-II. The thorough transformation of the absorption band of $\lambda_{\max } 407 \mathrm{~nm}$ into the band of $\lambda_{\max } 551 \mathrm{~nm}$ after the proton-acidifying process, which can be assigned to the prolonged periphery $\pi$ system as pathone show. And the Q-band become broader after the protonacidifying process, which can be assigned to the prolonged conjugated $\pi$ system of the central phthalocyanine' ring as path-two show. Considering the excellent stability of the central phthalocyanine' ring and together with the absorption spectra as show in Fig. 2, the mechanism of the acidifying process should be mainly followed the way of path-one.

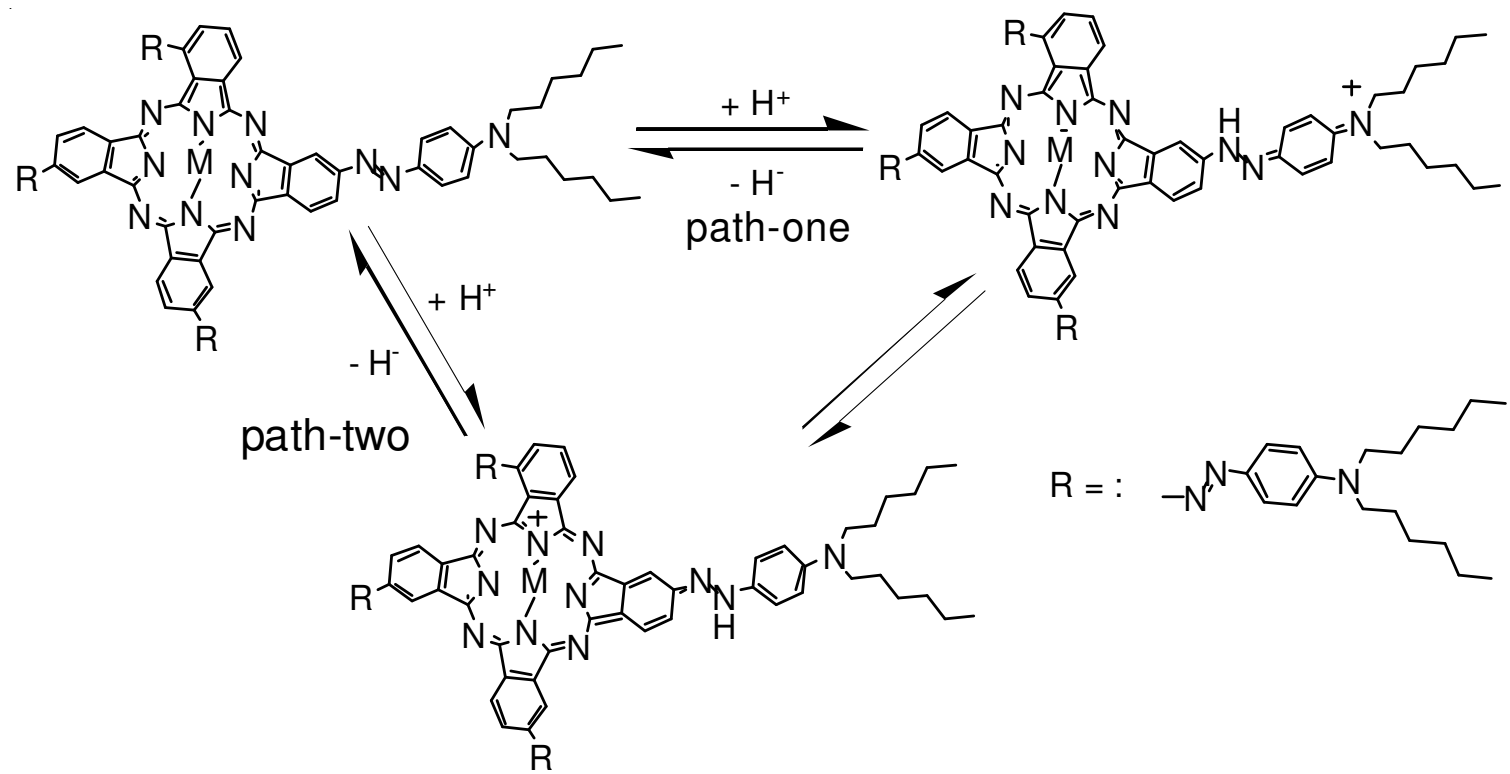

Scheme-II 
In order to provide a further understanding of this acidichromism process, a controlled experiment with the precursor c (3,4-dicyno-4'-N, N-dihexylaminoazobenzene) was carried out (Fig. 3). Fig. 3 shows the UV-VIS absorption spectra of the proton-acidifying process of the 20-layer Langmuir-Blodgett thin film of precursor $\mathbf{c}$ deposited on the substrate of quartz also under an average surface-pressure of $30 \mathrm{mN}$. Line 1 is the absorption spectrum of the LangmuirBlodgett thin film as prepared and line 2-7 shows the absorption spectra of the acidified thin film at different times (0-90 min) upon being set in air. Moreover, we found that its spectrum can also not return to its original pattern even after exposing the acidified thin film to gaseous $\mathrm{NH}_{3}$, which indicates an irreversible acidichromic process for the Langmuir-Blodgett thin film of the precursor $\mathbf{c}$. Therefore, it should be recognized that the reversible acidichromism for $\mathbf{a}$ is closely related to its molecular structure with phthalocyanine moiety. For compound b, its Langmuir-Blodgett thin films also show a reversible acidichromic behaviour similar to compound $\mathbf{a}$.

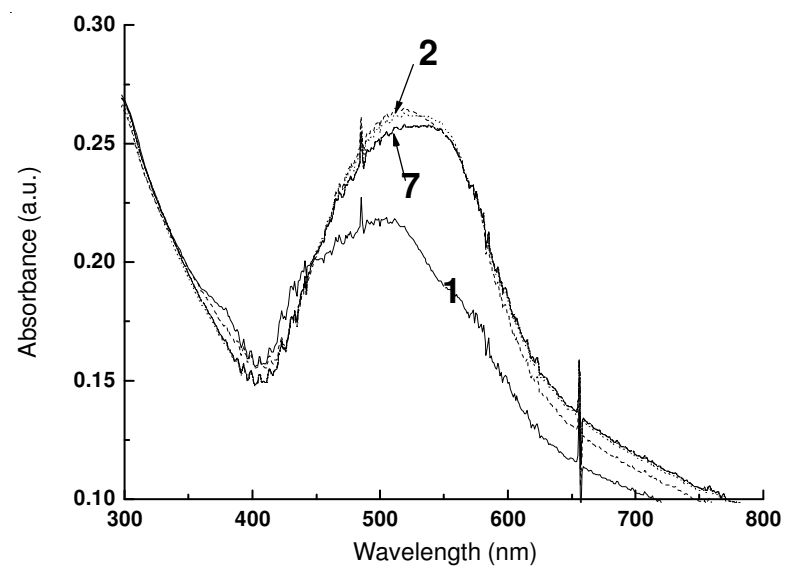

Fig. 3. UV-visible absorption spectra of the Langmuir-Blodgett film of the precursor $\mathrm{c}$ showing the irreversible acidichromism process

\section{Conclusion}

The Langmuir-Blodgett films of the azo group-modified phthalocyanines bearing longer alkyl groups on the periphery were successfully prepared. The acidichromism of the Langmuir-Blodgett thin films of compound $\mathbf{a}, \mathbf{b}$ and precursor c has been studied and the current work indicates that the phthalocyanine moiety in the molecule plays a very important role in the reversible acidichromic process. The fabricatability of them into Langmuir-Blodgett films makes it convenient for them to be fabricated into devices and the new properties might entrust some applications to these new phthalocyanines derivatives, such as fabricated into chemical sensor as well.

\section{REFERENCES}

1. C.C. Leznoff and A.B.P. Lever, (a) Phthalocyanines: Properties and Applications, VCH, New York, Vols. 1-4 (1989-1996); (b) N.B. Mckeown, Phthalocyanines: Synthesis, Sructure and Function, Cambridge Universty Press, New York (1998); (c) K.M. Kadish, K.M. Smith and R. Guilard, The Porphyrin Handbook, Academic Press, San Diego, Vols. 1-20 (2002-2003).

2. C.F. Van Nostrum, S.J. Picken, A.J. Schouten and R.M. Nolte, J. Am. Chem. Soc., 117, 9957 (1995).

3. J.M. Cook, N.B. Mckeown and A. Thompson, J. Chem. Mater., 1, 287 (1989).

4. G. Wegner, Mol. Cryt. Liq. Cryt., 235, 1 (1993).

5. M. Hanack, A. Beck and H. Lehmann, Synthesis, 703 (1987).

6. B.A. Gregg, M.A. Fox and A.J. Bard, J. Am. Chem. Soc., 111, 3024 (1989).

7. (a) J.H. Fuhrhop, C. Demoulin, C. Boettcher, J. Koning and U. Siggel, J. Am. Chem. Soc., 114, 4159 (1992); (b) J.H. Fuhrhop, U. Binding and U. Siggel, J. Am. Chem. Soc., 115, 11036 (1993).

8. A. Ulman, An Introduction to Ultrathin Oganic Film From LangmuirBlodgett to Self Assembly, Academic Press, Inc. San Diego, CA, p. 158 (1991) and references therein.

9. Y.-F. Li, S.-L. Li, K.-J. Jiang and L.-M. Yang, Chem. Lett., 33, 1450 (2004).

10. A.W. Snow and N.L. Jarvs, J. Am. Chem. Soc., 106, 4706 (1984). 